\title{
LARGE DEFLECTIONS OF HEAVY CANTILEVER BEAMS AND COLUMNS*
}

\author{
By ROBERT SCHMIDT (University of Detroit) \\ AND \\ DONALD A. DADEPPO (University of Arizona)
}

Large deflections of the heavy horizontal cantilever of uniform cross section have been investigated by Hummel and Morton [1], Bickley [2], and Rohde [3]. No exact analysis of the postbuckling deflections of the heavy column could be found in technical and mathematical publications. The purpose of this note is to extend and improve Rohde's results for the horizontal cantilever as well as to present an exact analysis of large postbuckling deflections of the heavy column. For notations refer to Fig. 1.

According to the Bernoulli-Euler hypothesis for slender beams and columns, the bending couple $M$ is proportional to the curvature $d \psi / d s$ of the deflected inextensional elastica. Hence,

$$
M=-E I \frac{d \psi}{d s},
$$

where $E I=$ const. is the flexural stiffness of the column and $d M / d s=w s \sin \psi$, by the conditions of equilibrium. Thus,

$$
\frac{d^{2} \psi}{d s^{2}}+\frac{w s}{E I} \sin \psi=0
$$

in which $w$ is the weight per unit length of the column. Let

$$
p=\left(\frac{w}{E I}\right)^{1 / 3} s, \quad \alpha=\psi(0), \quad T=\psi-\alpha ;
$$

then

and

$$
d^{2} T / d p^{2}+p(\sin \alpha \cos T+\cos \alpha \sin T)=0
$$

$$
T=\sum_{n=1}^{\infty} A_{3 n} p^{3 n}
$$

in which

$$
\begin{aligned}
& A_{3}=-(1 / 6) \sin \alpha, \quad A_{6}=-(1 / 30) A_{3} \cos \alpha, \\
& A_{9}=-(1 / 72)\left(A_{6} \cos \alpha-(1 / 2) A_{3}^{2} \sin \alpha\right), \cdots .
\end{aligned}
$$

Since $d x / d s=\cos \psi, d y / d s=\sin \psi$, Eqs. (3) and (5) yield

$$
\begin{aligned}
& x=(E I / w)^{1 / 3}(F \cos \alpha-G \sin \alpha), \\
& y=(E I / w)^{1 / 3}(F \sin \alpha+G \cos \alpha),
\end{aligned}
$$

\footnotetext{
- Received June 26, 1969.
} 


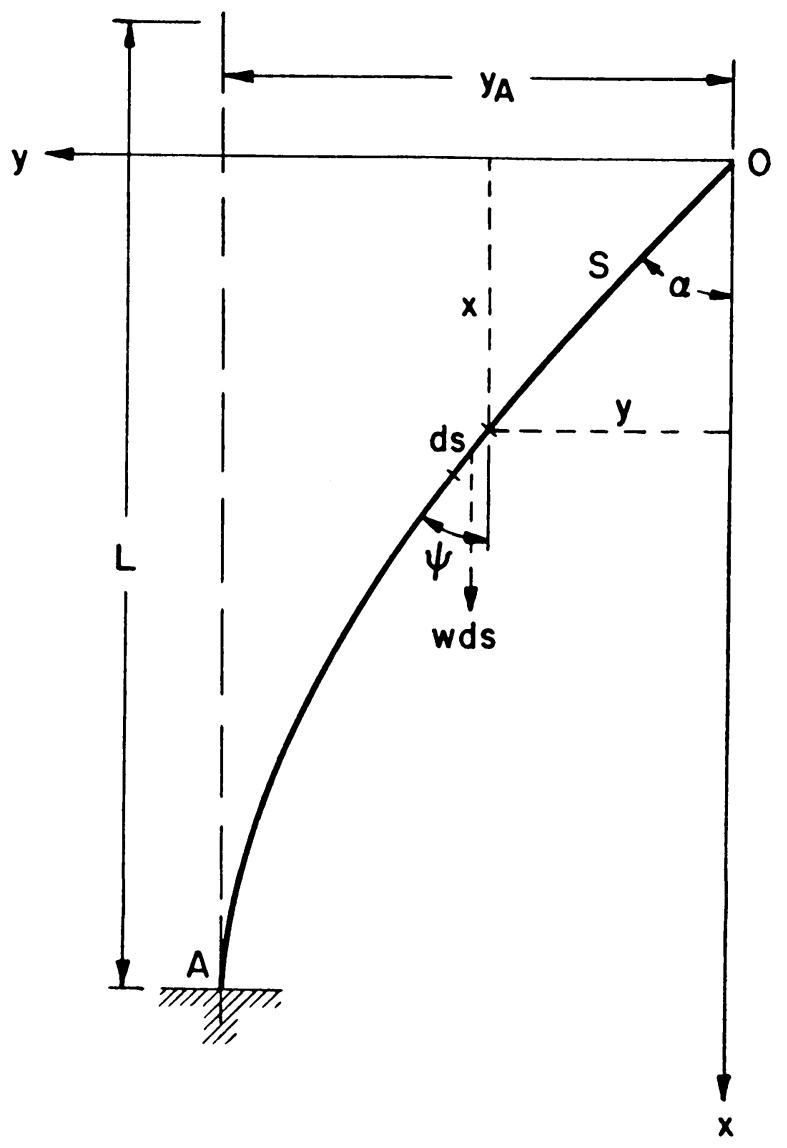

Fig. 1. Deformed heavy column.

where

$$
\begin{aligned}
& F=p-(1 / 14) A_{3}^{2} p^{7}-(1 / 10) A_{3} A_{6} p^{10}-\cdots \\
& G=(1 / 4) A_{3} p^{4}+(1 / 7) A_{6} p^{7}+(1 / 10)\left(A_{9}-(1 / 6) A_{3}^{3}\right) p^{10}+\cdots ;
\end{aligned}
$$

or, in view of Eqs. (2), (3), and (5),

$$
y=-\left(\frac{E I}{w}\right)^{1 / 3} \sum_{n=1}^{\infty} \frac{3 n(3 n-1)}{3 n-2} A_{3 n} p^{3 n-2} .
$$

The load $w$ is related to the free-end angle $\alpha$ by the condition $\psi=0$ at $s=L$, i.e., at the clamped end. Thus,

$$
\alpha=-\sum_{n=1}^{\infty} A_{3 n} \cdot\left(w L^{3} / E I\right)^{n}
$$

and, by reversion of this series,

$$
\begin{aligned}
\left(w L^{3} / E I\right) & =-\frac{1}{A_{3}} \alpha-\frac{A_{6}}{A_{3}^{3}} \alpha^{2}-\frac{1}{A_{3}^{5}}\left(2 A_{6}^{2}-A_{3} A_{9}\right) \alpha^{3}-\cdots \\
& =\frac{6 \alpha}{\sin \alpha}\left[1+\frac{\alpha}{5} \cot \alpha+\frac{\alpha^{2}\left(38-13 \sin ^{2} \alpha\right)}{600 \sin ^{2} \alpha}+\cdots\right] .
\end{aligned}
$$


By assuming values of $\alpha$ in Eq. (12) the corresponding values of $w$ are calculated. For $\alpha=0, w=w_{c r}=7.83735 E I / L^{3}$ is the buckling load in Euler's sense. For $\alpha>\pi / 2$ the convergence of series is not very satisfactory. The values of $w L^{3} / E I$ are plotted in Fig. 2 versus $\alpha / 2, y_{A} / L, 1-x_{A} / L$.

Fig. 3 presents improved extended results for the problem of the heavy horizontal cantilever considered by Rohde [3]. In addition to the exact solutions, the results obtained by means of a perturbation technique, based on the assumption

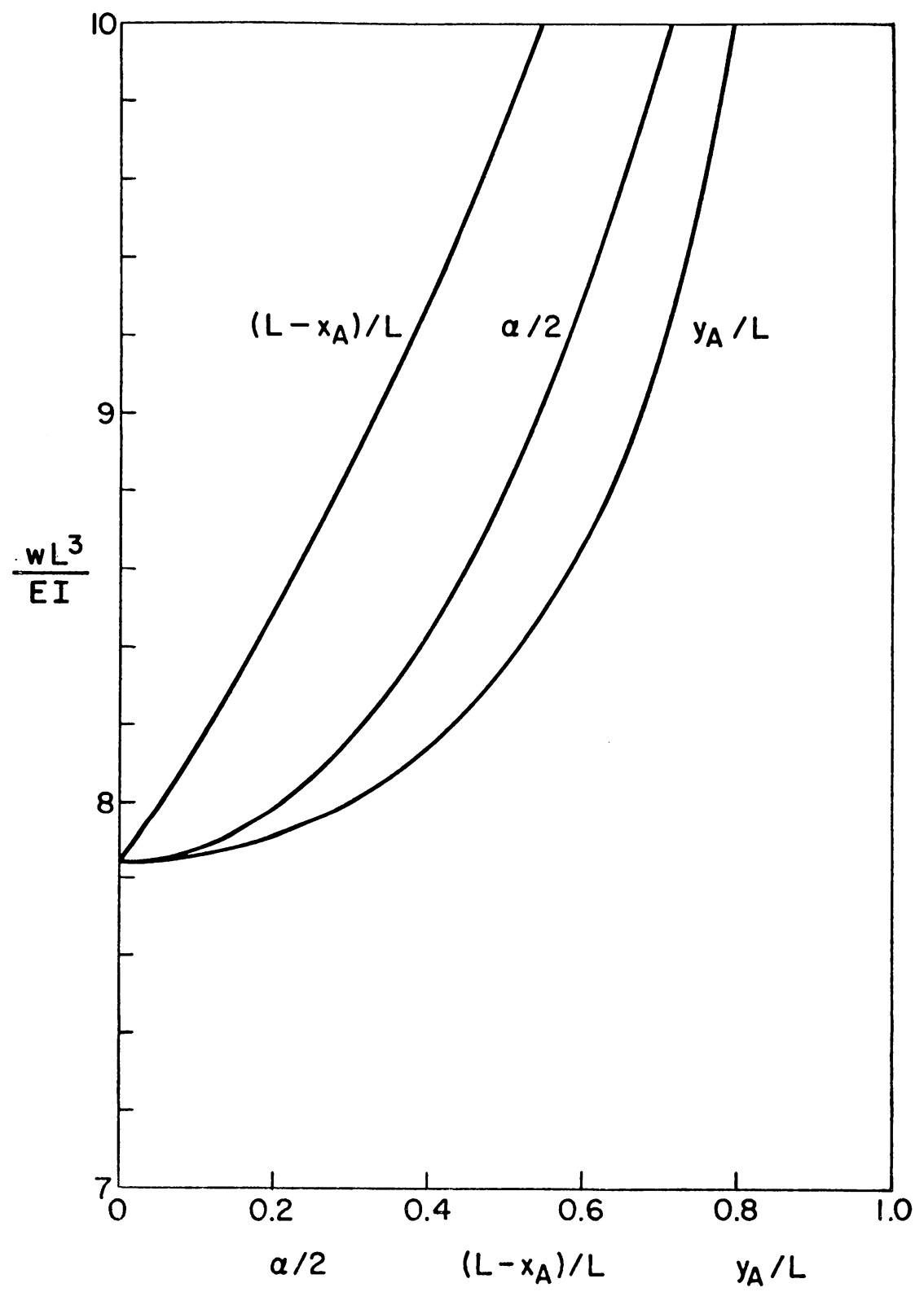

Fig. 2. Dimensionless load versus dimerisionless deflections of the free end of the heavy column. 


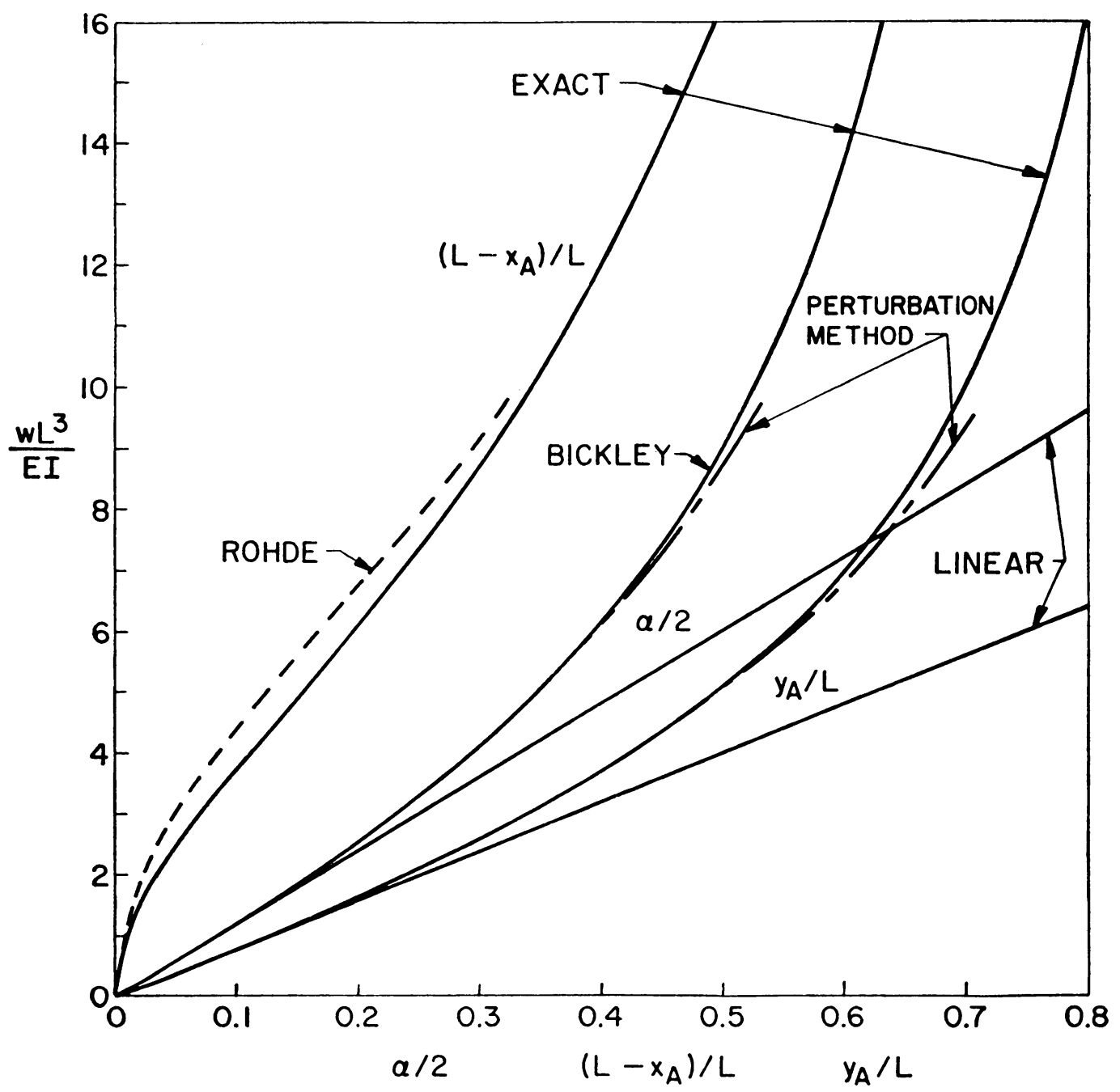

Fig. 3. Dimensionless load versus dimensionless deflections of the free end for the heavy horizontal cantilever.

$$
\psi=\psi_{1}(s) \alpha+\psi_{3}(s) \alpha^{3}+\psi_{5}(s) \alpha^{5}
$$

are also shown.

\section{REFERENCES}

1. F. H. Hummel and W. B. Morton, On the large bending of thin flexible strips and the measurement of their elasticity, Philos. Mag. (7) 4, 348-357 (1927)

2. W. G. Bickley, The heavy elastica, Philos. Mag. (7) 17, 603-622 (1934)

3. F. V. Rohde, Large deflections of a cantilever beam with uniformly distributed load, Quart. Appl. Math. 11, 337-338 (1953) 